\title{
Design of Patient Animal Heat Wireless Monitoring System Based on Zigbee
}

\author{
MA Lili \\ College of Automotive Engineering, Weifang University of Science and Technology, Shouguang, \\ China \\ e-mail: 44984858@qq.com
}

Keywords: ZigBee; wireless sensor network; CC2520; MSP430

\begin{abstract}
This design scheme for the wireless automatic monitoring network in health care system is proposed, and the software design of the coordinator node and sensor nodes, the network on how to use the ZigBee network technology and CC2520 RF chip design combined with wireless sensor networks have a certain coverage. In the medical field has higher use value.
\end{abstract}

\section{Introduction}

Now in most hospitals the patient care still use manual methods, in most cases, need to nurse according to bed numbers checked one by one, the efficiency is low,and encountered unexpected abnormal situations,easy for processing is not timely,resulting in medical accident.And now medical monitoring equipment generally using the cable way to transmit data,in the round the patient connection not only messy and affect the patient mood,so need wireless mode of a kind of low cost and high reliability of the produce.This paper mainly introduces the use of radio frequency chip CC2520 to realize the data acquisition, design of hardware and software and control system. The sensor node hardware design mainly completes the energy distribution of sensor nodes, the coordinator software mainly realize at the same time fusion monitoring and multiple zone data on a number of regional. The wireless medical monitoring system can realize the real-time remote monitoring of a plurality of patients, improve the work efficiency and safety. The telemedicine, an emerging strong discipline, stretches from the multi-disciplines of medicine, computer network, communication engineering.

\section{ZigBee Technology}

ZigBee technology is a kind of short distance wireless communication technology,the PHY layer and MAC layer protocol for the IEEE 802.15.4 protocol standard,the work of the ZigBee band $868 \mathrm{MHz}, \quad 915 \mathrm{MHz}$ respectively, $2.4 \mathrm{GHz}$ three band, including band $868 \mathrm{MHz}$ defines 1channel;frequency $915 \mathrm{MHz}$ defines 10 channel. Frequency $2.4 \mathrm{GHz}$ defines 16 channel.ZigBee technology adopts CSMA-CA channel access method,which can effectively avoid communication conflicts.The network layer protocol developed by the Zigbee alliance, ZigBee c devices can be constructed in a star network or a point-to-point network, connecting the 16 bits address short address and 64 bits long address is divided, with the larger network capacity. The application layer can be exploited according to the needs of users

\section{system design}

Monitoring system is mainly composed of individual monitoring terminal and internet remote server component,where in the individual monitoring terminal by physiological sensor nodes and embedded S3C2440 platform.Physiological sensor nodes by various types of physiological sensor module and Zigbee communication module,including various types of physiological sensor modules include temperature,heart rate,pulse module,they all have the characteristics of wearable,portable.Internet remote server by wireless router,internet public network and hospital 
control center,wireless router connected to the Internet public network through standard IEEE 802.11b protocol to realize data center,will be transferred to the hospital data terminal,to complete the whole process of care. The scheme of system as shown in figure 1.

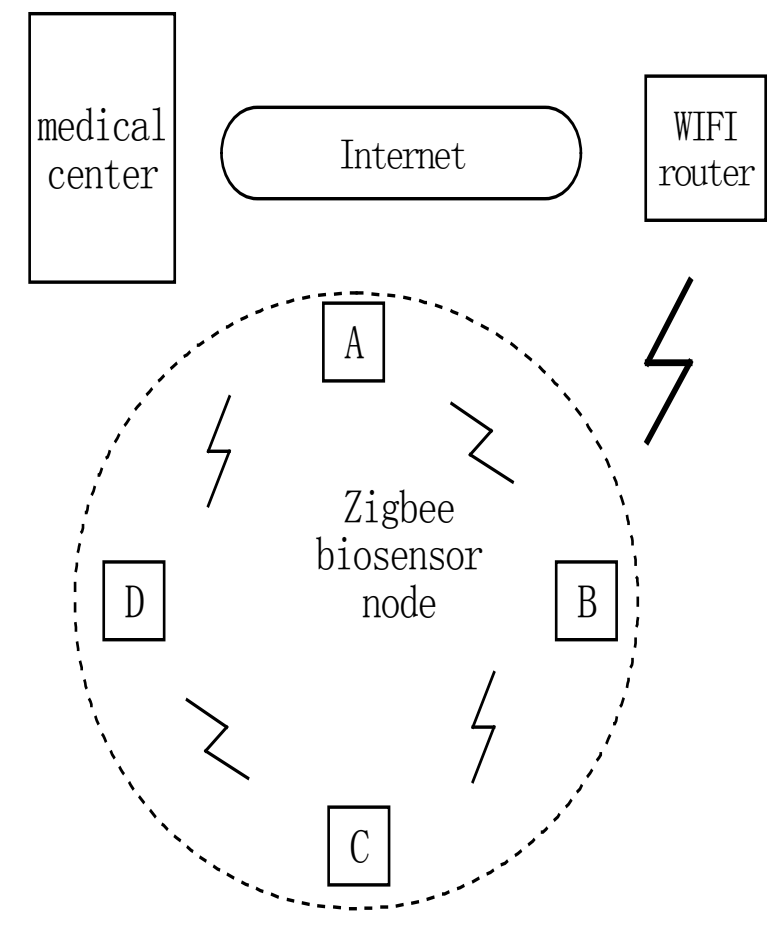

Figure 1. monitoring system schematic diagram

\section{ZigBee protocol}

The ZigBee protocol by the physical layer (PHY), medium access control (MAC) layer,network layer,application layer (NWK) (APL) and security service layer (SSP) five parts. The standard PHY layer and MAC layer by the 802.15.4 standard IEEE gives the definition of the NWK layer,MAC layer,APL layer and SSP layer,defined by the ZigBee alliance is given. Among them, the application layer contains the application support sub layer (Application Support Sublayer, APS )application framework (Application Famework,AF),ZigBee (Zigbee Device Objects device object, ZDO) and ZDO management platform. The Zigbee protocol frame as shown in figure 2. 


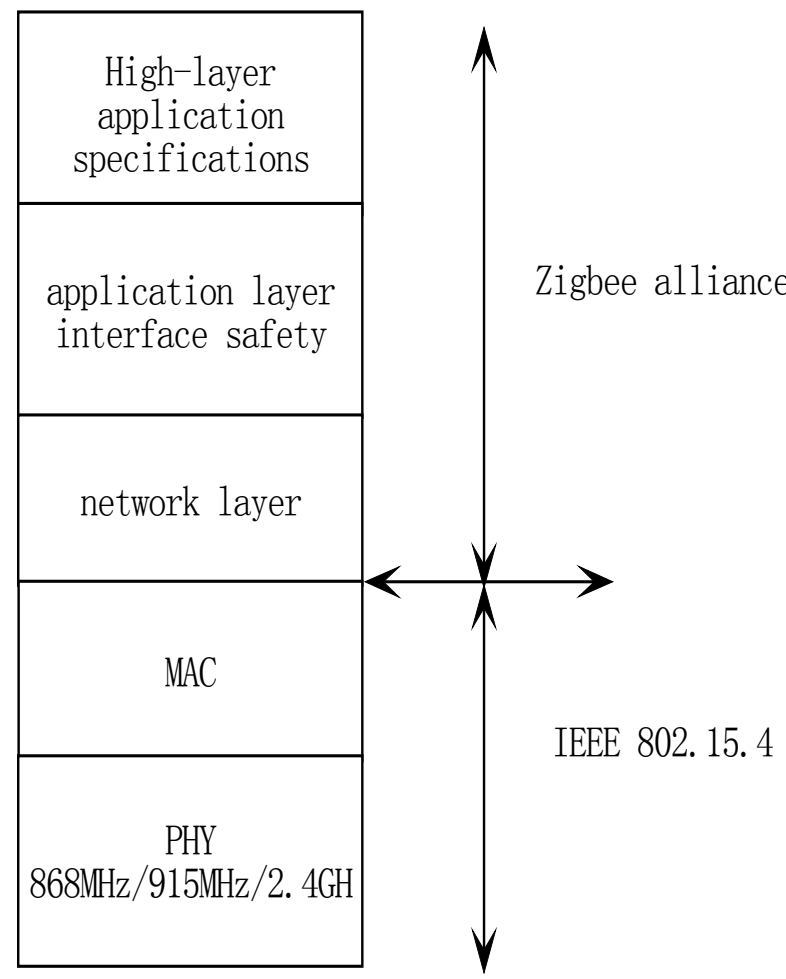

Figure 2. $\quad$ Protocol framework

\section{system hardware design}

In the system, monitoring the patient's self-organizing wireless sensor nodes form constitute a network through multi-hop relay data to the monitoring station will be monitored by the base station transmits data to the PC, the doctor or nurse can be connected PC to obtain the patient's physiological data, make timely treatment of care patients, mainly by monitoring base stations, wireless sensor nodes and routing nodes.

Personal monitoring terminal hardware components as shown in Figure 3,contains the main physiological sensor node,MCU MSP430 and S3C2440 embedded platform. Physiological sensor nodes by Chipcon company's CC2520 chip module and physiological sensor, CC2520 chip is a Zigbee RF (Radio Frequency) front-end,internal contains enhanced 8051 core,mainly used 2.4GHz IEEE802.15.4 standard and the ZigBee SOC(system System On a Chip, on-chip). Therefore, the physiological sensor nodes requires very few external components, without other chip, can meet the need of networking.S3C2440 embedded hardware platform is composed of S3C2440 main chip,WI-FI module, function keys,LCD display,USB,RS-232 and RJ45 of peripheral GPIO interface,S3C2440 main chip for Samsung Semiconductor Company production of ARM9 microcontroller using 16/32 RISC (Reduced Instruction Set Computer, reduced instruction set computer) of the ARM920T kernel, Harvard structure, ARMv6 instruction set processor and multimedia hardware accelerator. We successively in choosing its signal processing through the filter,amplification,etc. The research design.The pulse sensor module is based on S3C2440 ,by integrating Zigbee module and enhanced on the 8051 single chip and related peripheral circuits to implement, besides S3C2440 itself has excellent data processing ability, in the peripheral circuit design is also carefully selected made after weighing. 


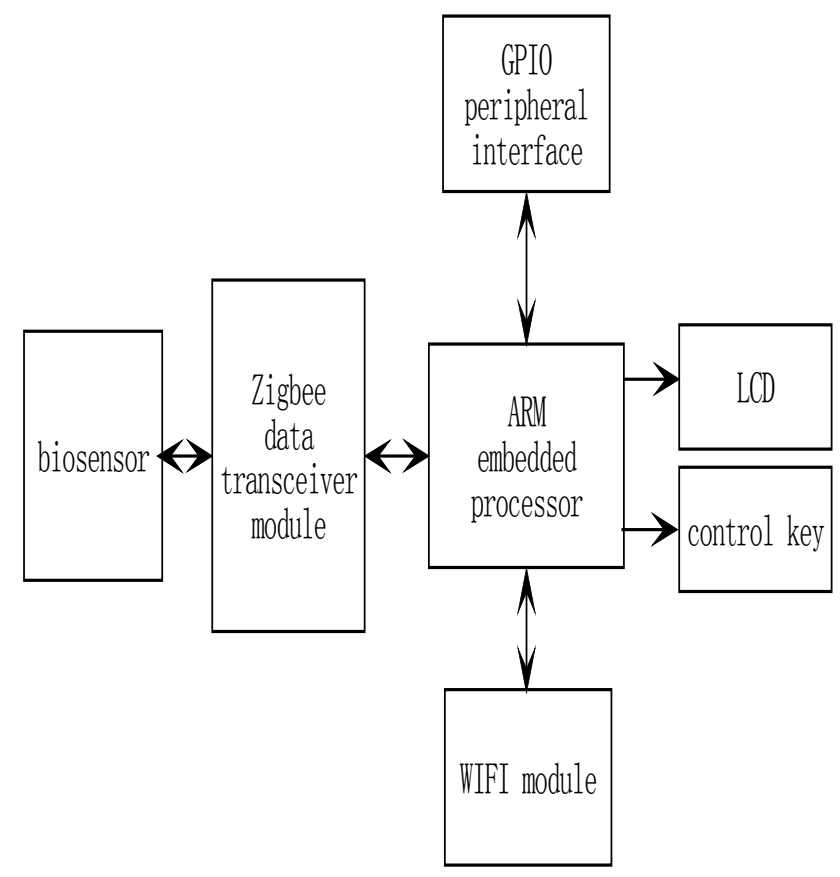

Figure 3. personal monitoring terminal hardware structure diagram

Physiological sensor nodes mainly by physiological sensor module and Zigbee wireless transmission module, as shown in figure 4. The circuit of HK-2000A pulse sensor in Hefei Huake company as sensor pulse sensor node, the output signal is synchronous analog 1.5V peak pulse signal.Pulse signal by the CC2520 on-chip ADC acquisition conversion, and on-chip ADC internal reference voltage is only $1.25 \mathrm{~V}$, in order to ensure the integrity of the pulse information, system adopts an external reference voltage is set, the AIN7 pin of the P0_7 port from the input $1.6 \mathrm{~V}$ voltage reference, where Vin is the output signal of the pulse sensor.

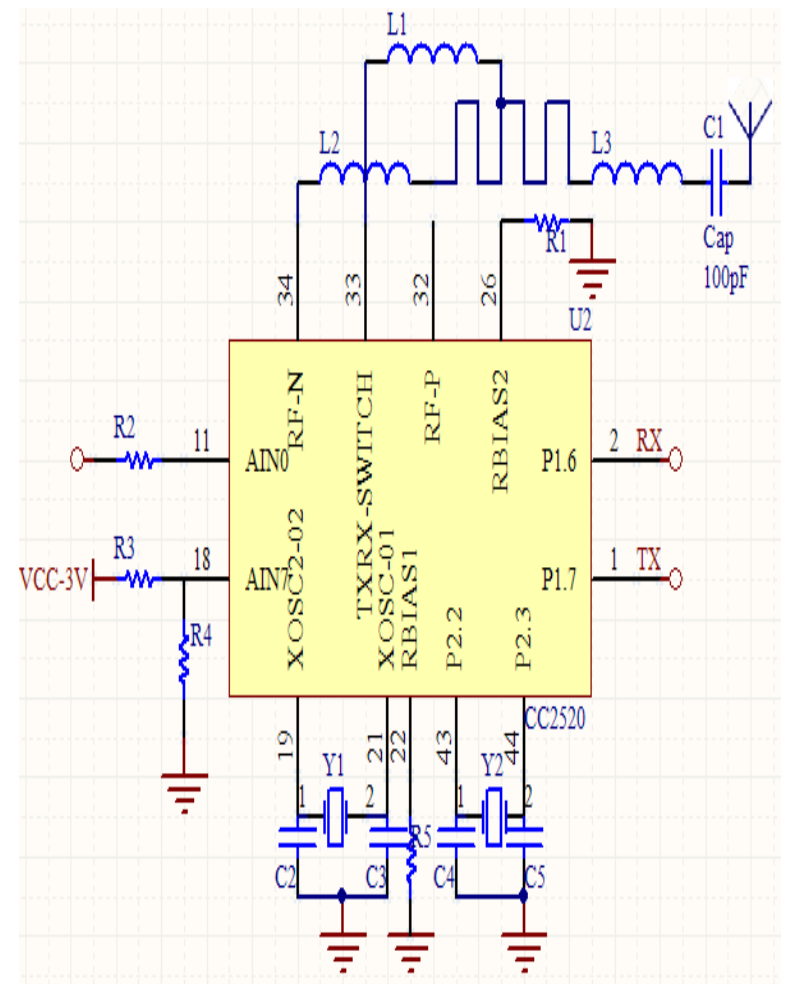

Figure 4. physiological sensor nodes 


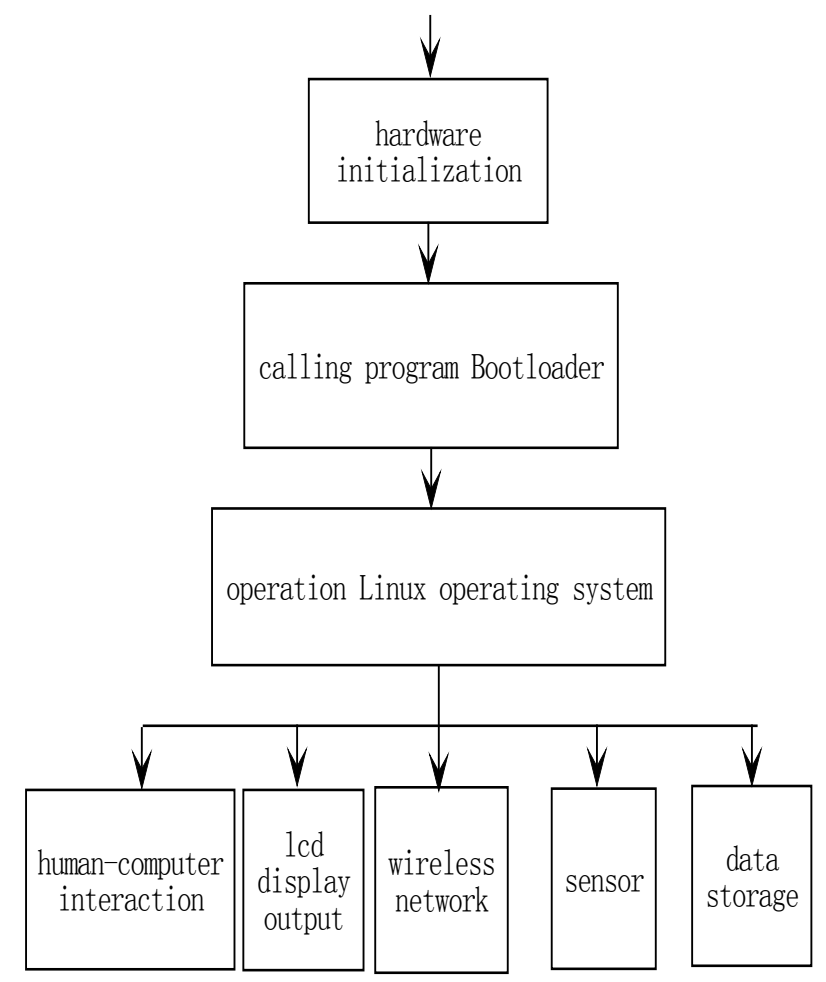

Figure 5. $\quad$ individual monitoring terminal operating system flow chart

Due to the design of mechanism of heart rate and body temperature sensor nodes and pulse sensor node is the same, they are adopting similar acquisition mode, therefore,in the design of the circuit is data acquisition port change.

\section{monitoring system software design}

Personal monitoring terminal operating system software by using Linux embedded operating system, is the operation mechanism of power system after loading the Boot loader system hardware port initialization, initialize the bottom of each hardware module, scheduling Boot loader, sets up the memory space,and the hardware and software environment of the system set to an appropriate state. Then the control right over to the operating system kernel boot program, operating system starts, the system according to the realization of the function, the whole system is divided into different levels of task.Personal monitoring terminal operating system process as shown in Figure 5, the system is powered on, firstly, hardware initialization, distribution of RAM and FLASH ROM space, and then call the Boot loader program, to load and start the Linux operating system. Start the Linux operating system is completed, the establishment of different tasks, the interface to establish communication message queue by the hardware support, the underlying hardware driver is loaded, execution out put display, man-machine dialogue, communication links, data acquisition and so on the different software task scheduling.

\section{Conclusion}

The design of the system has successfully realized the wireless acquisition of body temperature,pulse,ward indoor temperature and other information, the Zigbee module of the wireless network to transfer data to the monitoring center computer, realizes the application of wireless sensor network technology in medical monitoring system. The system can also be integrated a variety of sensor module, the realization of remote monitoring of a variety of information, in order to achieve for the monitoring of different patients. The system of fast data acquisition speed, low energy consumption, stable operation, and achieved the anticipated design goals of the system. 
The system uses a Zigbee module for data transmission, data transmission over wireless, simplify the work of laying the line, to create the conditions for large-scale practical application. PC program written in a flexible and feasible to break the shackles of LCD hardware conditions, the amount of information displayed, its graphic language more humane. A PC can simultaneously monitoring multiple patient's condition, due to constraints, we only had one team. If rises to the practical application, a corresponding set of equipment can be a patient, doctors and nurses can simultaneously monitoring multiple patients in the control room, reducing the burden on rounds.

\section{References}

[1] William Stallings, He Jun, etc.The wireless communication and network [M]. beauty: Tsinghua University press, 2005)

[2] LI Wenzhong - ZigBee wireless network technology introduction and actual combat [M]. Beijing: Beihang University press, 2007.

[3] PENG Yan, HE Jiandong. A System Based on Zigbee Technology to Monitor Ecoenvironment in Orchard[J]. Journal of Agricultural Mechanization Research, , 2009, 4(4): 164169.

[4] WU Fei,ZHANG Zhuxi, YANG Hongqiao. Design of Hospital Computer Room Wireless Monitor Aystem Based on Zigbee[J] Chinese Medical Equipment Journal,2008.29(12):55-58.

[5] GUO Shifu,MA Shuyuan,WU Pingdong,CHEN Zhilong. Pulse Wave Measurement System

Based on ZigBee Wireless Sensor Network[J] . Application Research of Computers,2007,24(4):258-260.

[6] ZigBee Alliance.ZigBee Speeification[DBOL].(2005-05-01)[2008-06-30].

[7] ZENG Shengtao.The design and implementation of remote mobile medical monitoring system based on internet of things, A Dissertation Submitted to Guangdong University of Technology for the Degree of Master,2014.05.

[8] ZHAO Ze,CUI Li.A RemoteHealth Care System Based onW ireless Sensor Networks, Information and Control,2006,02.

[9] YUAN Jiang. Design research based on CC2430 remote medical monitoring. A Dissertation Submitted to Xiamen University for the Degree of Master,2014.05.

[10] MAO Huanhuan. Design of medical care System Based on ZigBee. A Dissertation Submitted to Wuhan University of Technology for the Degree of Master,2013.05.

[11] Yan Fusong,Zhao Junhui,Li Xiuping. Zigbee technology and application. Guangdong Communication Technology, 2006.04

[12] Liu Wei. ECG Monitor System Research Based on Embedded ARM. A Dissertation Submitted to North China University of Technology for the Degree of Master,2014.06 